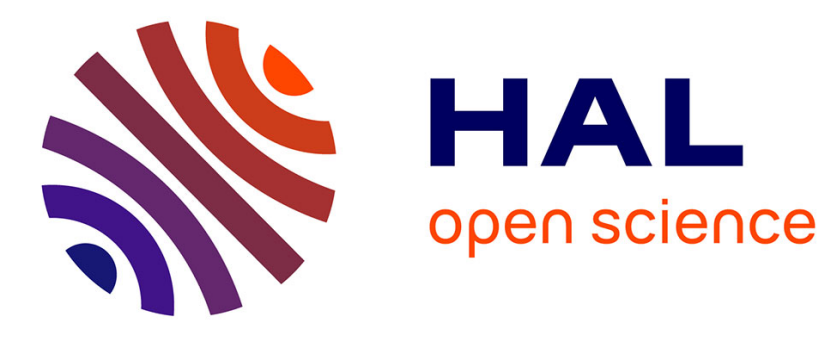

\title{
Layouten und Gestalten
}

Rodney Ast, Elodie Attia, Andrea Jördens, Christian Schneider

\section{To cite this version:}

Rodney Ast, Elodie Attia, Andrea Jördens, Christian Schneider. Layouten und Gestalten. Materiale Textkulturen. Konzepte - Materialien - Praktiken, , 2015, 10.1515/9783110371291.597 . hal01404823

\section{HAL Id: hal-01404823 \\ https://hal-amu.archives-ouvertes.fr/hal-01404823}

Submitted on 29 Nov 2016

HAL is a multi-disciplinary open access archive for the deposit and dissemination of scientific research documents, whether they are published or not. The documents may come from teaching and research institutions in France or abroad, or from public or private research centers.
L'archive ouverte pluridisciplinaire $\mathbf{H A L}$, est destinée au dépôt et à la diffusion de documents scientifiques de niveau recherche, publiés ou non, émanant des établissements d'enseignement et de recherche français ou étrangers, des laboratoires publics ou privés.

\section{(이)(\$)}

Distributed under a Creative Commons Attribution - NonCommercial - NoDerivatives| 4.0 
Rodney Ast (Papyrologie), Élodie Attia (Judaistik), Andrea Jördens (Papyrologie), Christian Schneider (Germanistik)

\section{Layouten und Gestalten}

Die meisten Schriftobjekte waren dazu bestimmt, gelesen zu werden, normalerweise von Menschen, gelegentlich auch von Göttern, manchmal von beiden. Ein irgendwie geartetes Publikum war - und ist - in jedem Akt des Schreibens implizit enthalten. Um eine Botschaft zu vermitteln und den Leseprozess ( $\rightarrow$ Lesen und Entziffern) zu erleichtern, konnte man sich bestimmter Konventionen bedienen. Dazu gehörten unter anderem der Gebrauch von Linien, gemalten Buchstaben, Satzzeichen oder Layout-Elementen. Dieser Artikel nimmt seinen Ausgangspunkt bei dem letztgenannten Merkmal, dem Layout des geschriebenen Wortes auf einem Trägermaterial. Er betrachtet die Weisen, in denen das Layout durch scheinbar disparate Faktoren wie ästhetische Erwägungen, praktische Beschränkungen, physische Eigenschaften, politische Ziele und funktionale Überlegungen beeinflusst werden konnte. Besonderes Gewicht wird auf das gelegt, was man sehr breit als die „westliche Tradition“ von der gräko-römischen Welt bis zum mittelalterlichen Europa bezeichnen könnte, wohlwissend, dass das Nachdenken über die Anordnung des geschriebenen Worts auf einer gegebenen Oberfläche etwas Universelles darstellt, das auch andere Räume und Kulturen beschäftigte.

In Griechenland taucht der früheste Beleg im 8. Jahrhundert v. Chr. auf, lange bevor eine Präferenz für das Schreiben von links nach rechts sich durchgesetzt hatte. Beeinflusst durch das phönizische Schreibsystem verlaufen die frühesten Inschriften (zumindest von unserem Blickwinkel aus) rückläufig von rechts nach links. Da die frühesten Inschriften meist nicht viel mehr als Besitzverzeichnisse darstellen sei es, dass sie menschlichen oder göttlichen Besitz anzeigen -, tendieren sie dazu, kurz zu sein. Sie beschränken sich auf größeren Objekten auf eine einzelne Zeile oder, bei kleineren Objekten wie Gefäßen oder Statuetten, auf ein Textband, das der Kontur der Objekte folgt. Längere Inschriftentexte schlängeln sich hin und her, von rechts nach links und dann von links nach rechts (oder umgekehrt). Dieser Stil heißt bustrophedon und hat seinen Namen von dem Bild des pflügenden Ochsen (gr. bus), der sich fortlaufend hin und her über ein Feld bewegt. ${ }^{1}$

Frühe Versuche, ein Layout zu erstellen, lassen sich an dem berühmten Becher von Pithekussai aus dem 8. Jahrhundert v. Chr., auch bekannt als „Nestorbecher“, feststellen (SEG [Supplementum Epigraphicum Graecum] XIV 604, CEG [Carmina

Dieser Beitrag ist im SFB 933 „Materiale Textkulturen“ entstanden, der durch die DFG finanziert wird.

1 Jeffery 1961, 43-50. 
Epigraphica Graeca] 454). Rückläufig geschrieben, sind die Verse auf drei abgesetzte Zeilen verteilt, von denen jede auf der rechten Seite beginnt und nach links verläuft. Der erste Vers - wenn es sich denn tatsächlich um einen Vers handelt - dient als eine Art Überschrift für die beiden darauffolgenden Hexameter. Zusammengenommen lauten die Verse: „Ich bin der Becher des Nestor, aus dem sich gut trinken ließ. Wer aber aus diesem Becher trinkt, den wird sofort das Verlangen nach der schönbekränzten Aphrodite ergreifen“. Obwohl die Verse auf das Objekt, auf dem sie sich befinden, bezogen sind, sind sie keineswegs ein integraler Bestandteil des Gesamtdesigns - man nimmt vielmehr an, dass es sich bei ihnen um einen späteren Zusatz handelt. Andere frühe Schriftbeispiele zeigen jedoch, wie das Layout des geschriebenen Wortes an der gesamten Szene teilhaben konnte. Die griechische Vasenmalerei bietet vielfältige Beispiele für dieses enge Miteinander von Text und Bild. Neben einem Bild angebrachte Inschriften können schlicht als Bezeichnung der dargestellten Figur - eines Gottes oder mythischen Helden - dienen. Jedoch bestehen sie in besonderen Fällen auch aus längeren Passagen. Ein gutes Beispiel dafür ist ein aus dem 6. Jahrhundert v. Chr. stammender kleiner Krug, ein sogenannter aryballos, aus Korinth. Er zeigt einen Doppelflöten-Spieler, der vor den Mitgliedern eines Chores steht. ${ }^{2}$ Während er sein Lied spielt, kommt aus seiner Flöte ein Hexameter-Vers hervor, der das Gefäß als Preis für Pyrvias, den Leiter des Chores, ausweist und sich unter- und oberhalb der Chormitglieder entrollt, von denen der erste (der Chorleiter selbst) in die Luft springt. In ähnlicher Weise stellt eine attische pelike - ein amphorenartiger Behälter - aus dem späten 6. Jahrhundert v. Chr., die in Vulci gefunden wurde und sich heute in der Eremitage in St. Petersburg befindet, drei Figuren dar, zwei sitzend, eine stehend, die himmelwärts nach einer über ihnen schwebenden Schwalbe sehen. ${ }^{3}$ Worte fließen aus jedem ihrer Münder hervor und imitieren so Sprache. In diesen Fällen greift die Schrift, so wie sie auf dem Gefäß angebracht ist, in die Bildszene ein und steigert die Komplexität der Abbildung.

Auch in Monumentalinschriften konnte das Layout den allgemeineren Zwecken des Objekts dienen. Eine attische Grabinschrift für einen gewissen Autokleides (IG I ${ }^{3}$ 1273bis) wohl aus dem 6. Jahrhundert v. Chr. bietet hierfür ein gutes Beispiel: Nicht nur sind die Verse rückläufig geschrieben, sondern auch von unten nach oben, offensichtlich um die Worte so darzustellen, als würden sie von dem Verstorbenen gesprochen (Abb. 1).

Sehr frühe griechische Schriftbeispiele zeigen eine bemerkenswerte Vielfalt der Darstellung, worin sich vielleicht lokale Traditionen widerspiegeln. Allmählich jedoch bildeten sich verbindliche Konventionen heraus, die geschriebene Texte einer gewissen formalen Einheitlichkeit unterwarfen. Das zeigt sich besonders an Monumentalinschriften, in denen Überschriften, Zwischenüberschriften, Absätze, Spalten

2 Wachter 2001, 44, 47 und 281.

3 Immerwahr 2009, CAVI 7346. 


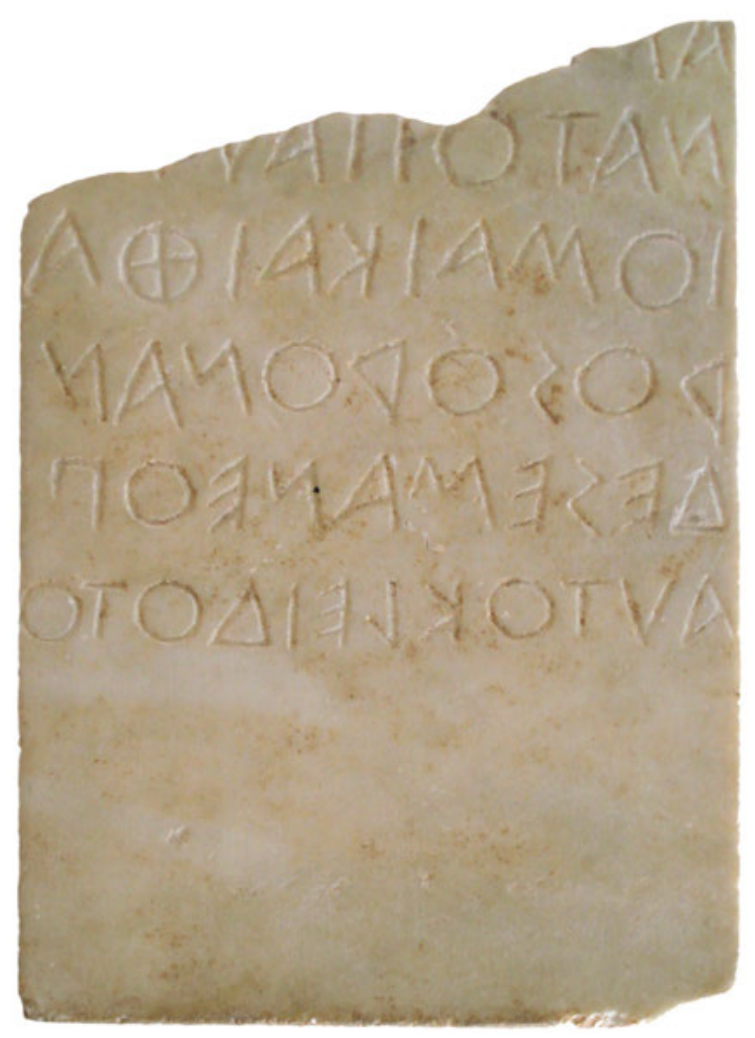

Abb. 1: Grabstele des Autokleides IG I³ 1273bis; wohl 6. Jahrhundert v. Chr. (৫ Julia Lougovaya).

und regelmäßige Zeilenabstände schließlich wichtige Bestandteile des Layouts werden. In einem frühen Stadium der Entwicklung von Layoutkonventionen etabliert sich in Athen ein Stil, der gut zwei Jahrhunderte Bestand haben sollte. Bekannt ist er als stoichedon-Schreibweise (gr. stoichos „Reihe“ oder „Spalte“). Der Name bezieht sich auf die strenge, gitterförmige Anordnung der Zeichen auf der Schreiboberfläche, wo ein Buchstabe in einer geraden, vertikalen Reihe über den anderen gesetzt wird ( $\rightarrow$ Beurkundungen, Abb. 2). Eine seltene und erst kürzlich entdeckte Variation dieses Stils ist das plinthedon - von gr. plinthos „Ziegel(-stein)“ -, ein ziegelartiges Arrangement von abwechselnden stoichedon-Zeilen. ${ }^{4}$ Das Aufkommen des stoichedon im späten 6. oder frühen 5. Jahrhundert fällt mit einer steigenden Zahl öffentlich ausgestellter Inschriften zusammen, wie etwa Dekreten, Tribut- oder Gefallenenlisten. Der Vorteil dieses Layouts liegt darin, dass es der Inschrift eine regelhafte, unhinterfragbare Ordnung vermittelt und gleichzeitig dem gesamten Ensemble von Text und Objekt eine höhere Monumentalität verleiht. Ein Nachteil ist, dass dieses

4 Vgl. SEG LV 413. 
Layout bestimmten Schreibkonventionen, die gleichfalls als Lesehilfe gedacht sind, zuwiderläuft, zum Beispiel der Interpunktion und der Worttrennung zwischen den Zeilen (vgl. z. B. die Marmorstele AA XIX P 5 [367/66 v. Chr.] $\rightarrow$ Beurkundungen, Fallbeispiel 2).

Wenn man nicht nur inschriftliche Quellen, sondern auch andere Medien wie Papyri ( $\rightarrow$ Papyrus) und Ostraka $(\rightarrow$ Ton) berücksichtigt, stellt man ein breites Spektrum von Layoutkonventionen fest, die für verschiedene Texttypen genutzt wurden. $\mathrm{Zu}$ den Faktoren, die die Wahl eines Layouts bestimmen, zählen insbesondere Lesbarkeit ( $\rightarrow$ Lesen und Entziffern), rasche Texterfassung sowie eine ästhetisch ansprechende Gestaltung. Bei literarischen Texten zum Beispiel kann der abwechselnde Einsatz von eisthesis and ekthesis (Einrückung nach rechts bzw. Ausrückung nach links) den Wechsel des Metrums oder Sprechers (oder beides) signalisieren oder auf das Vorliegen eines bestimmten Verstyps hinweisen, etwa auf den Pentameter in der Elegie. Auch in nichtliterarischen Papyri kann das Layout ein wirksames Mittel sein, das Genre eines bestimmten Textes zu identifizieren. Eine Überschrift, gefolgt von einer Namensliste zur Linken und Ziffern zur Rechten einer Seite, deutet sofort auf eine Abrechnung hin. Alphabetisch geordnete Aufstellungen von Wörtern, die in Silben unterteilt sind, kennzeichnen eine Silbentabelle - eine verbreitete Schulübung, die sich das ganze römische Ägypten hindurch findet. Auch mathematische Übungen, wie Multiplikationstabellen, folgen oft einem festgelegten Layout: Multiplikator, Multiplikand und Produkt erscheinen in drei Spalten auf derselben Linie angeordnet. So kann das Lernen durch das Layout erleichtert werden. Wie sehr Fragen des Layouts andererseits auch gesellschaftlichen Konventionen unterliegen, die sich im Laufe der Zeit durchaus verändern können, zeigt sich namentlich im Bereich des Briefverkehrs ( $\rightarrow$ Papyrus, Fallbeispiel Papyrusbriefe).

In offiziösen Kontexten finden wir ebenfalls fixe Layouts. Eine große Kanzleischrift in Latein zum Beispiel, die oben und unten ein im Übrigen griechisches Dokument rahmt, ist typisch für spätantike Gerichtsprotokolle. Zusammen mit der Schrift verleiht das Layout dem Dokument auf diese Weise Autorität, indem es als eine Art offizielles Siegel fungiert.

Wie funktionale Gesichtspunkte das Layout eines Artefakts bestimmen, wird besonders an kleinen Keramikscherben deutlich, wie sie sich überall in der ägyptischen Großen Oase (Oasis magna) gefunden haben. Die Scherben waren als Etiketten in die Verschlüsse von Krügen eingelassen, um die wichtigsten Informationen hinsichtlich des Inhalts der Krüge zu verzeichnen, nämlich das Lieferjahr, die Herkunftsangabe und den Namen der für die Lieferung verantwortlichen Person. Diese Informationen wurden prägnant und fast immer in derselben Reihenfolge „eingeschrieben“, so dass das Layout ein wichtiger und schnell identifizierbarer Bestandteil des Etiketts war. Ein anderes bemerkenswertes Beispiel für die Funktionsgebundenheit eines Layouts findet man in der sogenannten Sechszeugendoppelurkunde, die in Ägypten und dem Nahen Osten seit hellenistischer Zeit bis weit in die hohe Kaiserzeit hinein belegt ist (vgl. z. B. P. Cair. Zenon I 59003 [259 v. Chr.] $\rightarrow$ Beurkundungen, Fall- 
beispiel 3), oder auch in den zweisprachigen Urkunden aus den dörflichen Staatsnotariaten der frühen Römerzeit, den sogenannten Grapheia. Quer unter den demotisch gefassten, auf zwei Kolumnen verteilten Partien der Geldbezahlungs- und Abstandsschrift befindet sich hier die sogenannte hypographe des Grapheionschreibers, in der noch einmal der Vertragstext in griechischer Sprache wiedergegeben ist und die außerdem die - eigenhändige oder durch Dritte vollzogene - Unterschrift der Vertragsparteien sowie einen Registrierungsvermerk enthält, wodurch die früheren Zeugenlisten auf der Rückseite entbehrlich wurden. ${ }^{5}$ Ohnehin haben sich gerade für Verträge in wohl allen Kulturen feste Layoutgewohnheiten herausgebildet, was zunächst zweifellos Sicherungszwecken diente und zudem den Wiedererkennungswert erhöhte ( $\rightarrow$ Beurkundungen). Für noch wichtiger dürfte freilich einzuschätzen sein, dass man dem Artefakt damit zugleich in spezifischer Weise $\rightarrow$ Bedeutung zuschrieb, die auch dem, der es nicht in die Hand nahm, auf den ersten Blick begreiflich war.

Fast jeder identifizierbare Texttyp weist ein mehr oder weniger typisches Layout auf. Unterschiede hinsichtlich des Layouts ergeben sich dabei zuallererst aus der Natur des schrifttragenden Artefakts als solchem. Gefäße, $\rightarrow$ Textilien, Papyri, Pergament- oder Papiercodices ( $\rightarrow$ Pergament, $\rightarrow$ Papier, $\rightarrow$ Zwischen Pergament und Papier) lassen schon aufgrund des verwendeten Beschreibstoffes nicht dieselbe Art und Weise der Beschriftung zu. Wenn wir noch einen Blick auf die Codices werfen, die sich seit der Zeitenwende im Mittelmeerraum verbreiteten und seit dem 7. Jahrhundert n. Chr. auch von Arabern und Juden übernommen wurden, so zeigt sich, dass das Layout - insbesondere Seitenbild und Schriftspiegel - vor allem durch die Liniierung bestimmt werden (Abb. 2). ${ }^{6}$

Für die Liniierung wurden senkrechte Linien zur beidseitigen Begrenzung des Schriftraums gezogen sowie waagerechte Linien zur oberen und unteren Begrenzung. Auf diese Weise wurde der Schriftraum in eine, zwei, gelegentlich auch drei oder mehr Spalten eingeteilt. Waagerechte Linien im Inneren des Schriftraums markierten die Zeilen. Sämtliche Linien wurden durch Einstichlöcher vorbereitet. Diese Punkturen wurden an den Ober- und Unterrändern für die senkrechten Linien und am Außen-, gelegentlich auch am Innenrand für die waagerechten Zeilen in regelmäßigen Abständen vorgenommen. Sie dienten als Endpunkte für die mit dem stumpfen Ende des Griffels (sogenannte Blindzeilen) oder auch einem weichen Metallstift auf das Blatt aufgebrachten Begrenzungslinien und Zeilen. ${ }^{7}$ Die hierdurch angelegte Struktur verweist bereits auf die materielle Verwendung des schrifttragenden Artefakts, da dokumentarische Schriftstücke wie etwa Briefe üblicherweise nicht liniiert wurden. Ein Codex wurde hingegen liniiert, und zwar je nach der beabsichtigten Verwendung. So erhielt beispielsweise ein persönliches Buch nur eine Rahmung, während aufwen-

5 Eindrucksvolle Beispiele hierfür jetzt bei Lippert u. Schentuleit 2010.

6 Vgl. zum Folgenden ganz allgemein das Glossar von Jakobi-Mirwald $2008^{3}$.

7 S. dazu Schneider $2014^{3}, 128-133$. 


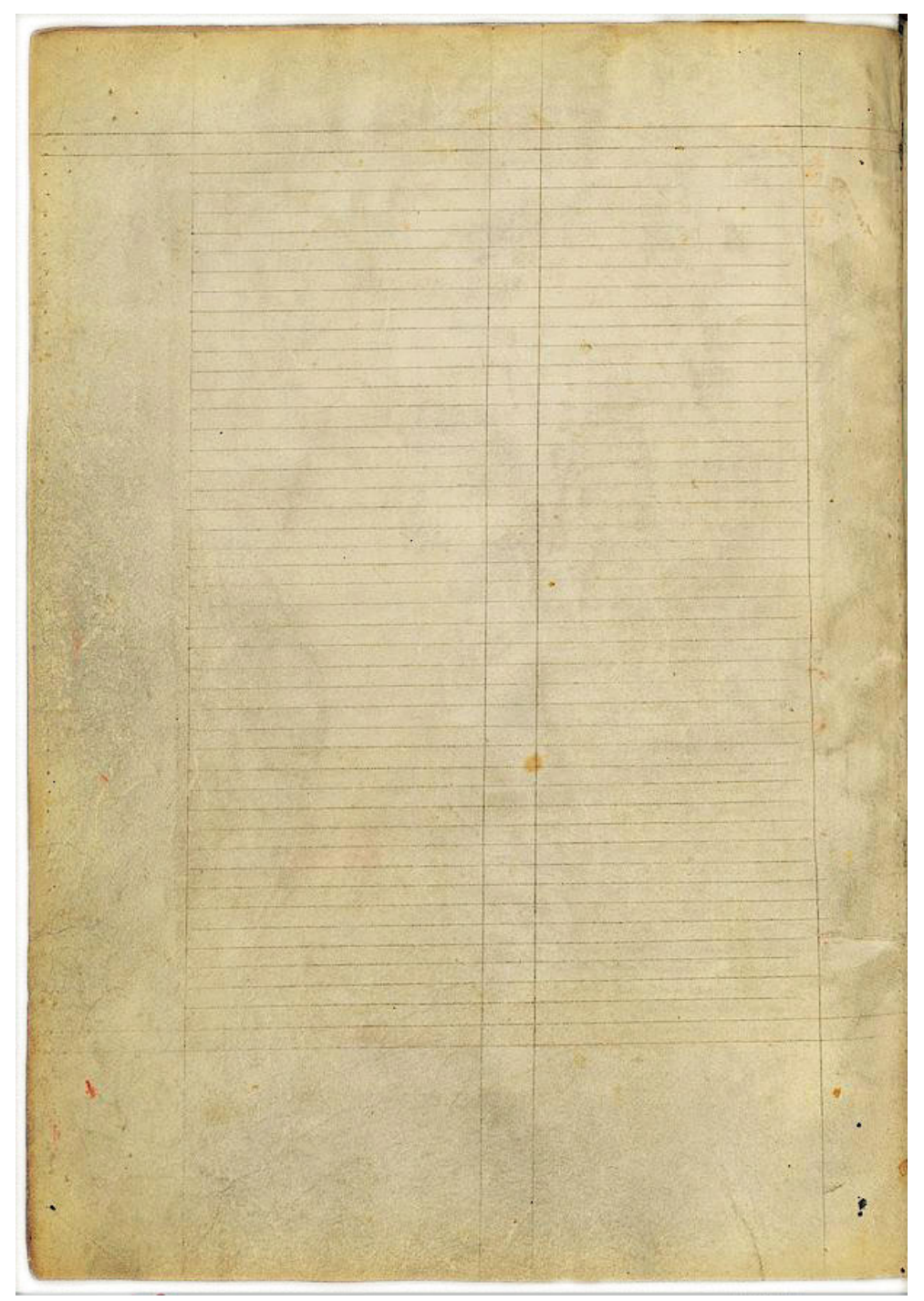

Abb. 2: Typisches, zweispaltiges Layout einer mittelalterlichen Handschriftenseite (Codex Manesse, fol. 109v); am linken Seitenrand noch gut zu erkennen die Einstichlöcher für eine gleichmäßige Liniierung (@ Universitätsbibliothek Heidelberg, Cod. Pal. germ. 848, fol. 109v).

digere Liniierungen wie zusätzliche senkrechte und waagerechte Linien stets von den besonderen Bedürfnissen der mise en page und mise en texte ihren Ausgang nahmen. Im Fall der hebräischen Bibel etwa ist die Liniierung besonders komplex, weil sie den beschriebenen Bereich des biblischen Textes abgrenzt, aber auch den seiner Masora 
oder der zugehörigen Kommentare. Zentrum, Ränder und Interkolumnien werden also bereits durch die Liniierung vorgegeben.

Als mise en page bezeichnet man die Anordnung des Geschriebenen auf der Seite. Sie ist beim Manuskript eng an das Schema der Liniierung gebunden, die ihr vorausgeht und sie bestimmt. Diese Anordnung variiert je nach Texttyp - profan, sakral, literarisch, als Kommentar, als Dokument (Brief, Heiratsvertrag, offizielle Verordnung, Gesetz). Es kann ein, zwei oder drei Spalten geben, auf Rändern können Kommentare oder erläuternde Notizen stehen. Manuskripte in zwei oder mehreren Sprachen haben dagegen eine andere mise en page. Mitunter kann es eine Hierarchisierung der Texte geben. So ist beispielsweise die hebräische Bibel sehr häufig von einer aramäischen Übersetzung begleitet, dem sogenannten Targum. Dieser Targum kann verseweise angeordnet sein, so dass stets auf jeden hebräischen Vers ein aramäischer folgt, aber auch in einer eigenen Spalte, und dann einem Hierarchisierungsprozess unterliegen. ${ }^{8}$

Die mise en texte umfasst nach einer Bestimmung des Literaturwissenschaftlers und Komparatisten Roger Laufer all jene Elemente, die zu einer Gliederung des Textes beitragen, um die Auffindung bestimmter Stellen zu erleichtern und dem Leser Unterstützung bei der Verwendung des beschriebenen Gegenstands zu bieten. ${ }^{9}$ Dies betrifft etwa Absätze, Paragraphen, Überschriften, Majuskeln, Versalien oder Initialen. So zeigt sich, dass Layoutkonventionen, die zu verschiedenen Zeiten an unterschiedlichen Orten entstanden - ob in literarischen, sakralen oder Alltagstexten -, spezifischen, manchmal auch mehreren Zwecken dienen: von der Erleichterung des Leseverstehens, dem Schutz der Privatsphäre, der Inhaltsbestimmung bis hin zur Kundgabe der Autorität derjenigen Stelle, die ein Dokument herausgab. Zugleich verweisen Layoutkonventionen auf lokale Traditionen. Als kulturelle Techniken verraten sie uns viel über die Grundsätze derjenigen $\rightarrow$ Textkultur, aus der sie hervorgegangen sind.

\section{Fallbeispiel 1: Das Grabepigramm des Sophytos (ausgehendes 1. Jahrhundert v. Chr./1. Jahrhundert n. Chr.)}

Für freistehende Grab- und Ehreninschriften hatte sich in der griechischen und römischen Antike im Laufe der Zeit ein relativ festes Format herausgebildet. Gern dienten sie als Statuenbasis oder trugen ein Relief. In diesem Fall konnten sie auch mit einem Giebel bekrönt sein, der von einer einfachen Rahmung über einen Dreiecksgiebel bis

8 Attia 2014.

9 Laufer 1983. 
hin zu akroterartigen Aufsätzen reichte. In der Regel waren sie hochrechteckig, nicht selten war auch das eigentliche Schriftfeld gerahmt. Die Schrift lief grundsätzlich von Rand zu Rand, wenngleich einzelne Wörter - Namen oder Begriffe - auch einmal betont auf Mitte gesetzt wurden, wie es vor allem in späterer Zeit auch Variationen im Schriftgrad gab. Dieses Prinzip wurde nicht einmal bei Versen durchbrochen. Zwar wurde der Text gelegentlich zentriert oder eingerückt, doch bilden größere Freiränder oder auch ein Flatterrand, weil Vers und Zeile übereinstimmen sollten, eher die Ausnahme.

Veränderungen gab es am ehesten bei den Formaten der Inschriften, da dies in besonderem Maße von Aufstellungsort und -zweck, lokalen Traditionen, persönlichen Vorlieben und schließlich auch Moden abhängig war. Bei Ehreninschriften, die eine Statue trugen, handelte es sich zumeist um hüfthohe Blöcke von quadratischem Grundriss, bei Grabinschriften häufiger um Stelen von einiger Tiefe. In beiden Fällen konnten auch die Schmalseiten, in besonderen Ausnahmen sogar auch die Rückseiten beschriftet sein. In Wände eingelassene Grabplatten wiesen dagegen unbearbeitete Rückseiten auf und hatten in der Regel auch geringere Dimensionen. Insbesondere bei denjenigen Grabplatten, die als Verschlüsse der sogenannten loculi - für die Aufnahme des Leichnams geschaffener Hohlräume - dienten, setzte sich allmählich ein Querformat durch.

Bei allen Varianten und Entwicklungen des Erscheinungsbildes, das durch die jeweils gewählten verschiedenen Schrifttypen noch zusätzliche Akzente erhielt, handelte es sich gleichwohl um ein relativ einheitliches Format. Dies zeigt der Vergleich mit einer 2004 im heute afghanischen Kandahar gefundenen griechischen Inschrift, deren Layout in jeder Hinsicht einzigartig erscheint (Abb. 3). Die üblicherweise als „Grabepigramm des Sophytos“ vorgestellte Inschrift aus dem damaligen Alexandria in Arachosien besteht aus zehn Distichen mitsamt Überschrift und einem auf der linken Seite leicht abgesetzten Akrostichon, das unter Wiederholung der Anfangsbuchstaben einer jeden Zeile auf den Namen des Auftraggebers Sophytos, den Sohn des Naratos, verweist. Dem Namen nach sicher indischer Herkunft, legt Sophytos darin Zeugnis von seinem Lebensschicksal ab - dem Verlust von Eltern, Heimat und Vermögen, jahrzehntelangem Exil, erfolgreicher Handelstätigkeit und schließlich der Heimkehr, wo ihm der Wiederaufbau des einst Verlorenen gelang. Die nahezu perfekt erhaltene, auf der Rückseite unbearbeitete Platte dürfte in eine Wand eingelassen gewesen sein, doch ist über das Aussehen des Monuments als Ganzes wie auch über den Aufstellungsort nichts mehr in Erfahrung zu bringen. Der mittig über den Text gesetzte Titel „Des Sophytos Stele“ und das Akrostichon sind gegenüber dem Haupttext leicht vergrößert. Auffällig ist auch das großzügige Interlineum, das durchschnittlich 1,75 cm hoch ist und damit fast die doppelte Höhe der etwa $1 \mathrm{~cm}$ hohen Textzeilen besitzt. Bereits von den Erstherausgebern wurde die Nähe der Schrift zu Buchschriften hervorgehoben, die sich vor allem aus den lunaren, „mondsichelförmigen“ 


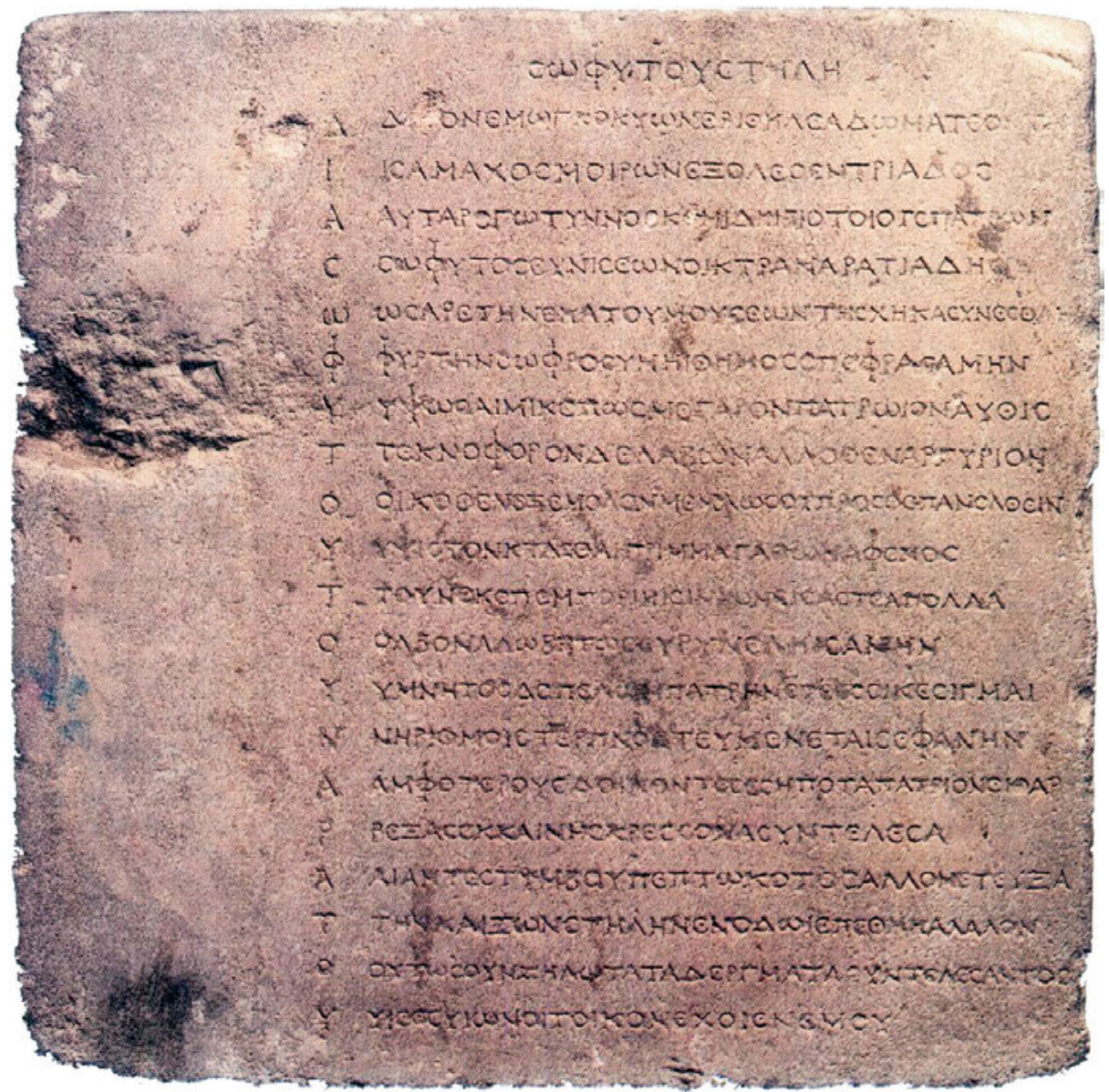

Abb. 3: Grabepigramm des Sophytos; ausgehendes 1. Jahrhundert v. Chr./1. Jahrhundert n. Chr. (SEG LIV 1568) (CC BY-NC-ND Bernard u. a. 2004, 230 fig. 1).

Formen von Sigma, Epsilon und Omega ergibt. ${ }^{10}$ Hatte man den Text anfangs übereinstimmend in das spätere 2. Jahrhundert v. Chr. gesetzt, ließen weitere paläographische Details zuletzt sogar eine Neudatierung in das ausgehende 1. Jahrhundert v. Chr. oder gar die frühe Kaiserzeit erwägen. In dieselbe Richtung weist eine sorgfältige inhaltliche Analyse, da die nächsten strukturellen Parallelen zu dem ungewöhnlich breiten Lebensbericht bemerkenswerterweise in den griechischen Grabinschriften

10 Die Kurzcharakteristik ,imitating cursive script of manuscripts“ in SEG LIV 1568 ist allerdings missverständlich, da es sich eindeutig um eine „gebaute“ Schrift ohne jeden kursiven Zug handelt. 
von Angehörigen der einheimischen Eliten Ägyptens zu finden sind. ${ }^{11}$ So mag Sophytos einer der Händler gewesen sein, die die um die Zeitenwende wiederauflebenden Beziehungen zwischen Indien und Ägypten nutzten, und das Gedicht eben dort in Auftrag gegeben haben. Der Steinmetz, dem er nach seiner Rückkehr das Blatt mit dem Text in die Hand drückte, hatte vermutlich noch nie eine griechische Grab- oder Ehreninschrift gesehen. Daher kopierte er die Vorlage detailgetreu, und zwar sowohl, was die mise en texte, als auch, was die mise en page betrifft. Denn mit ihren leicht gerundeten Ecken, der quadratischen Form und zumal dem ausgesprochen breiten Freirand auf der linken Seite, wodurch die mittig über den Text gesetzte Überschrift seltsam aus dem Zentrum verschoben erscheint, wirkt die Inschrift als nichts anderes als ein übergroßes Codexblatt.

\section{Fallbeispiel 2: Mikrographien in aschkenasischen Manuskripten: Eine besondere Form der mise}

\section{en page}

Die Masora der Hebräischen Bibel ist ein Apparat zum Bibeltext, der seit dem 8./9. Jahrhundert n. Chr. in Handschriften in Codexform auftaucht. Dieser Apparat, der durch Masoreten aus Sorge um die korrekte Niederschrift und Aussprache des geoffenbarten Textes entwickelt wurde, kommt nur in den mit diakritischen Zeichen Vokalzeichnen und Akzenten - ausgestatteten Bibeln vor. Die mittelalterlichen Bibelcodices folgen im Ganzen der mise en page und mise en texte des konsonantischen Textes der Torarollen, die für den liturgischen Gebrauch geschrieben werden, ${ }^{12}$ aber sie haben komplexere Formen des Layouts entwickelt. ${ }^{13}$ Der masoretische Apparat besteht aus zwei Teilen: Aus der masora parva, die üblicherweise am Kolumnenrand angebracht ist, und aus der masora magna, die auf den oberen und unteren Seitenrand platziert ist. Die besondere mise en texte dieses Apparates bedingt die Verwendung winziger Buchstaben, die einem Drittel oder Viertel der Größe des Haupttextes entsprechen, was diese Mikrographie kennzeichnet. ${ }^{14}$ In der Folgezeit fanden Veränderungen bei der mise en texte der Masora statt. So ist bereits im 9. Jahrhundert in den Handschriften, die aus dem Mittleren Osten stammen, $\mathrm{zu}$ beobachten, dass

11 Dazu demnächst Lougovaya (im Druck); dort auch die frühere Literatur.

12 Vgl. die Traktate Megillah und Soferim des babylonischen Talmuds. Der biblische Text von Exodus 15,1-18 z. B. muss in einer statt in drei Spalten geschrieben werden und dazu noch in einem ganz spezifischen Layout („Schachbrettmuster“).

13 Der masoretische Apparat wird im Talmud nicht erwähnt. Deswegen sind die Bibelcodices, die wir heute kennen, spezifische historische Artefakte des Mittelalters; vgl. Olszowy-Schlanger 2012.

14 Halperin 2013. 


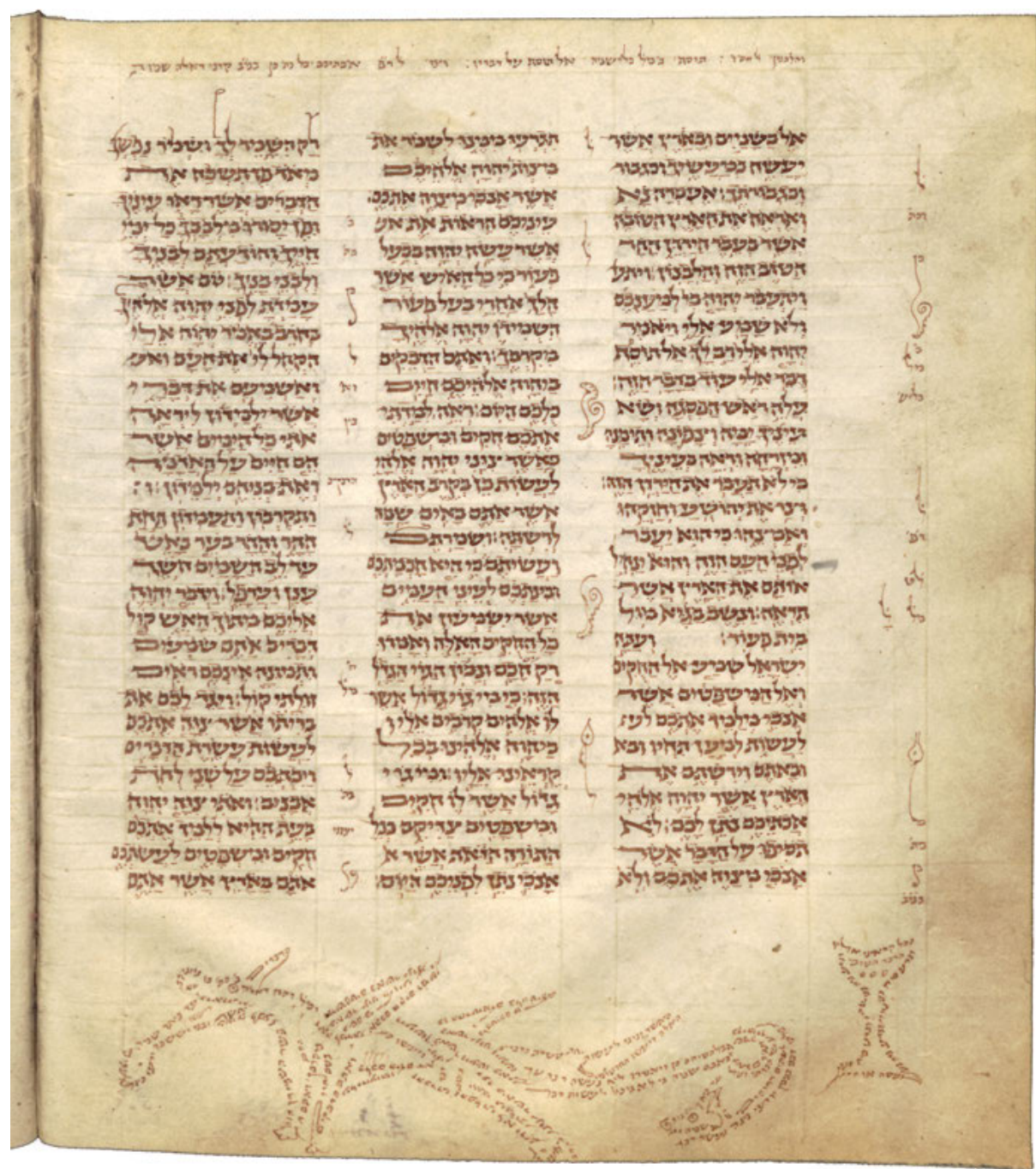

Abb. 4: Eine Mikrographie in der Form eines Drachen zu Deut 3:4-4:14, einer Passage, der zufolge figürliche Repräsentationen von Menschen und Tieren verboten sind $(\odot$ Staatsbibliothek zu Berlin - Preußischer Kulturbesitz, Orientabteilung MS Or. Qu. 9, fol. 110v).

der mikrographische Text dekorative oder architektonische Formen annehmen kann (siehe z. B. die Handschrift der National Library of Russia, St. Petersburg, EBP II B 17 und den sogenannten Codex Leningradensis). ${ }^{15}$

15 Beit-Arié u. a. 1997, 53-65, 114-131. 
Doch später, im 13. Jahrhundert, entwickelte sich in Spanien und in Westeuropa eine ganz besondere Darstellungsweise der Masora: Sie wird ornamental (d.h. geometrisch) oder figurativ (d. h. gegenständlich) ausgestaltet, indem darin ein Objekt, ein Ort oder ein Lebewesen - Menschen und reale oder fantastische Tiere - dargestellt werden (Abb. 4). In diesem Fall erscheint also die ursprüngliche mise en texte, wo in zwei oder drei Zeilen die masora magna geschrieben wird, modifiziert. Für diesen kulturellen Wandel der Schreibertradition gibt es bislang ebenso wenig eine Erklärung wie für das Auftreten der ornamentalen und figurativen Masora. Ist sie, wie es heute zumeist gesehen wird, ein Beleg für die Unkenntnis der Bedeutung des masoretischen Apparats - also für ein mangelndes philologisches Verständnis -, oder handelt es sich eher um die Entwicklung einer anderen Art von Verständigung, nämlich für mnemotechnische, pädagogische oder exegetische Zwecke, wie die $\rightarrow$ Edition der figurativen Masora in Ms. Vat. Ebr. 14 vermuten lässt? ${ }^{16}$ Eine systematische Untersuchung der materiellen Charakteristika der spätmittelalterlichen hebräischen Bibeln bleibt noch zu leisten.

\section{Literaturverzeichnis}

Attia (2014): Élodie Attia, „Targum Layouts in Ashkenazi Manuscripts. Preliminary Methodological Observations“, in: Adina Houtman, Eveline van Staalduine-Sulman u. Hans-Martin Kirn (Hgg.), A Jewish Targum in a Christian World (Jewish and Christian perspectives series 27), Leiden, 99-122.

Attia (im Druck): Élodie Attia, The Masorah of Elijah ha-Naqdan: An Edition of Ashkenazi Micrographical Notes (Materiale Textkulturen 11), Berlin/München/Boston 2015.

Beit-Arié u. a. (1997): Malachi Beit-Arié, Colette Sirat u. Mordechai Glatzer, Monumenta Palaeographica Medii Aevi. Codices hebraicis litteris exarati quo tempore scripti fuerint exhibentes, Bd. 1: Jusqu'à 1020 (Monumenta palaeographica medii aevi. Series Hebraica 1), Turnhout.

Bernard u. a. (2004): Paul Bernard, Georges-Jean Pinault u. Georges Rougemont, „Deux nouvelles inscriptions grecques de l'Asie centrale“, in: Journal des savants, 227-356.

Halperin (2013): Dalia-Ruth Halperin, Illuminating in Micrography. The Catalan Micrography Mahzor-MS Hebrew $8^{\circ} 6527$ in the National Library of Israel (The Medieval and early modern Iberian world 51), Leiden.

Immerwahr (2009): Henry R. Immerwahr, Corpus of Attic Verse Inscriptions, http://www.unc. edu/ hri/, mirror site http://avi.unibas.ch/home.html (Stand 29.4.2014)

Jakobi-Mirwald $\left(2008^{3}\right)$ : Christine Jakobi-Mirwald, Buchmalerei. Terminologie in der Kunstgeschichte, Berlin.

Jeffery (1961): Lilian H. Jeffery, The Local Scripts of Archaic Greece. A Study of the Origin of the Greek Alphabet and Its Development from the 8th to the 5th Centuries b. C. (Oxford Monographs on Classical Archaeology), Oxford.

16 Hierzu und zu weiteren Beispielen vgl. Attia (im Druck). 
Laufer (1983): Roger Laufer, „L'espace visuel du livre ancien“, in: Henri-Jean Martin u. Roger Chartier (Hgg.), Histoire de l'édition française, Bd. 1: Le livre conquérant: du Moyen Âge au milieu du XVIle siècle, 479-497.

Lippert u. Schentuleit (2010): Sandra L. Lippert u. Maren Schentuleit, Demotische Dokumente aus Dime III: Urkunden, Wiesbaden.

Lougovaya (im Druck): Julia Lougovaya, „Greek Poetry in a Post-Greek Milieu: The Epigram for Sophytos from Kandahar Contextualized“.

Olszowy-Schlanger (2012): Judith Olszowy-Schlanger, „The Hebrew Bible“, in: Richard Marsden u. E. Ann Matter (Hgg.), The New Cambridge History of the Bible, Bd. 2: From 600 to 1450, Cambridge, 19-40.

Schneider (2014 $\left.{ }^{3}\right)$ : Karin Schneider, Paläographie und Handschriftenkunde für Germanisten. Eine Einführung (Sammlung kurzer Grammatiken germanischer Dialekte, B. Ergänzungsreihe 8), Berlin/Boston.

Wachter (2001): Rudolf Wachter, Non-Attic Greek vase inscriptions, Oxford. 
Brought to you by | Universite Mediterranee-Aix Marseille II

Authenticated Download Date | 11/29/16 11:51 AM 